\section{Stabilized images: Further evidence for central pattern processing*}

\section{R. BROWN, M. J. SCHMIDT, M. P. COSGROVE, and J.J. ZUBER Purdue University, West Lafayette, Ind. 47907}

This experiment combined selective adaptation conditions with stabilized retinal images in order to provide support for the hypothesis that whole pattern fading of images reflects the operation of supraretinal pattern processing units. Pattern preadaptation was shown to enhance or suppress complete image fading when compared to light adaptation. The effects were demonstrated both when a single eye was adapted and tested and when one eye was adapted and the other was tested. This was taken as strong support for the notion that whole fades reflect pattern processing beyond the retina.

These studies further develop our attempts to account for stabilized retinal image phenomena and to examine their utility as a tool for identifying and describing pattern analytic processes in the human visual system. In earlier papers in the series, the suggestion that the fragmentation of pattern elements in stabilized view could be accounted for in terms of cortical pattern processing units has been supported (Brown, Schmidt, Fulgham, \& Cosgrove, in press; Cosgrove, Schmidt, Fulgham, \& Brown, 1971, 1972; Schmidt, Cosgrove, \& Brown, 1972; Schmidt, Fulgham, \& Brown, 1971).

In one of these studies (Schmidt, Cosgrove, \& Brown, 1972), it was demonstrated that adaptation to a nonstabilized grating stimulus produced orientation-specific effects on the fading of stabilized line stimuli. The results were in general agreement with earlier psychophysical (e.g., Campbell \& Kulikowski, 1966; Fidell, 1970) and neurophysiological (e.g., Hubel \& Wiesel, 1965, 1968) data that have indicated that pattern processing mechanisms may be specific to orientations within a range of 5-15 deg. The study reported herein was designed to extend these results by converging adaptation operations and stabilized image techniques in monoptic and dichoptic viewing. OBSERVERS

Two males, 24 (M.C.) and 25 (M.S.) years of age, served as Os. Vision was corrected to $20 / 20$ in the left viewing eyes with individually fitted contact lenses worn during all experimental sessions. Both Os were well practiced in stabilized image viewing.

$$
\text { STIMULI }
$$

Three adapting fields were used: (1) a light-filled circular field, (2) a circular field with horizontal black grating bars on a light background, and

* This research was supported by Research Grant HD-00909 from the National Institute of Child Health and Human Development.
(3) a circular field with vertical black grating bars on a light background. In each case, field diameter was $5 \mathrm{deg}$ $12 \mathrm{~min}$. Grating bars were parallel, $2 \mathrm{~min}$ in width, and separated by $10 \mathrm{~min}$ of light background. Adapting gratings of these dimensions were selected in order to equalize test line width and grating bar width, while at the same time creating a periodic pattern of 5 cycles/deg.

The test stimulus for all sessions appeared as a vertical black line segment $2 \mathrm{~min}$ in width and $50 \mathrm{~min}$ in length, centered in a circular (5 deg
12 min ) light-filled field.

\section{OPTICAL SYSTEM}

An optical system similar in principle to that described by Clowes \& Ditchburn (1959) was modified to include two channels, each capable of presenting the $\mathrm{O}$ with an image in either stabilized or nonstabilized Maxwellian view. This system is shown schematically in Fig. 1. Essentially, stabilization was achieved by means of an optical lever reflected from a small mirror fixed to a contact lens worn on the viewing eye. Nonstabilized Maxwellian view in the same system was obtained by shifting the O's biteboard laterally, allowing the primary projection path to pass directly through the O's pupil, thus aroiding the contast lens mirror and optical lever.

Contact lenses were scleral lenses individually molded to fit each $\mathrm{O}$. A small stage $(5-\mathrm{mm}$ diam $)$ was glued to the lens on the temporal side of the corneal bulge; a circular first-surface mirror (4-mm diam) was mounted on the stage normal to the optical axis.

Shutters operated by stepping motors were located in each channel to permit selection of either channel. Mean field luminance under all viewing conditions was adjusted to a measured

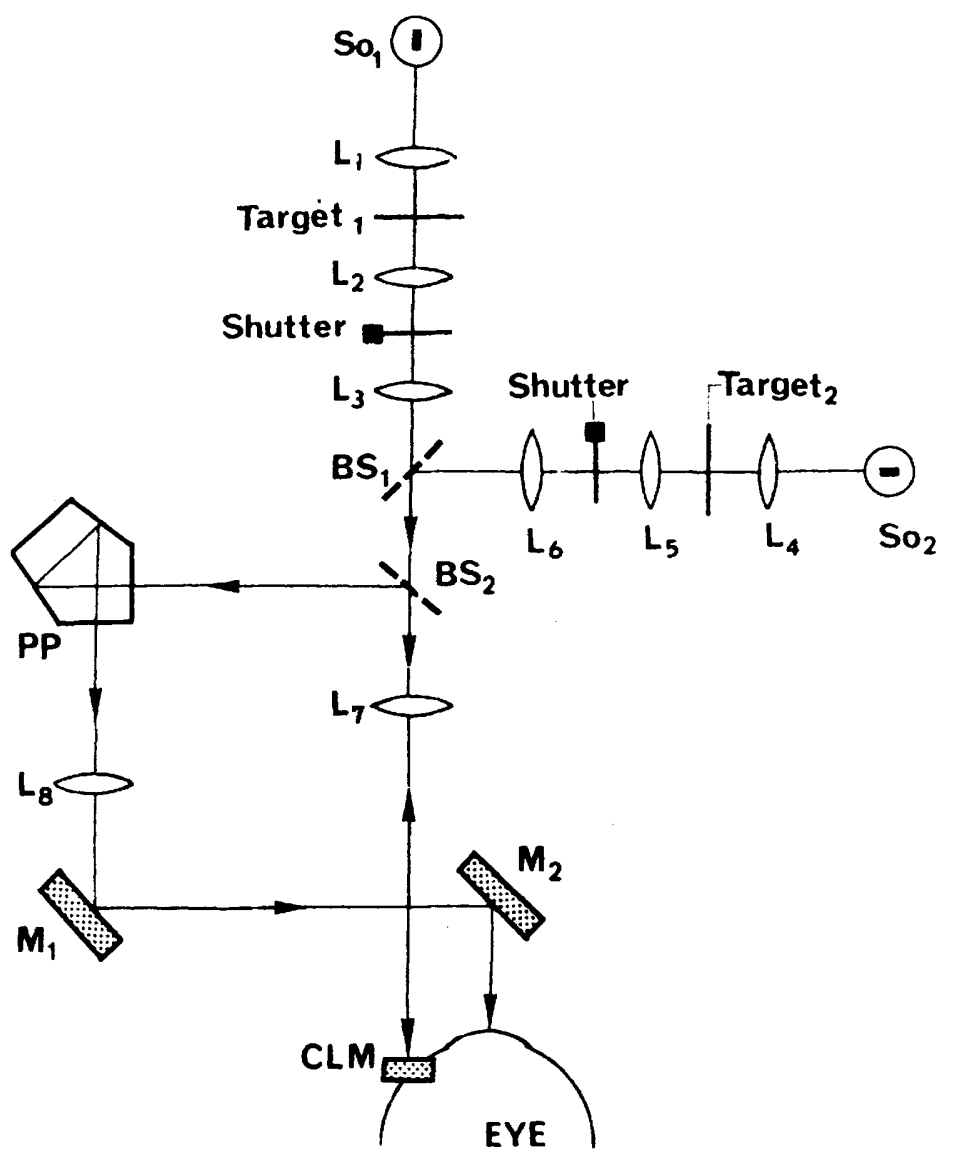

Fig. 1. Schematic diagram of the optical system. ${ }^{1}$ 


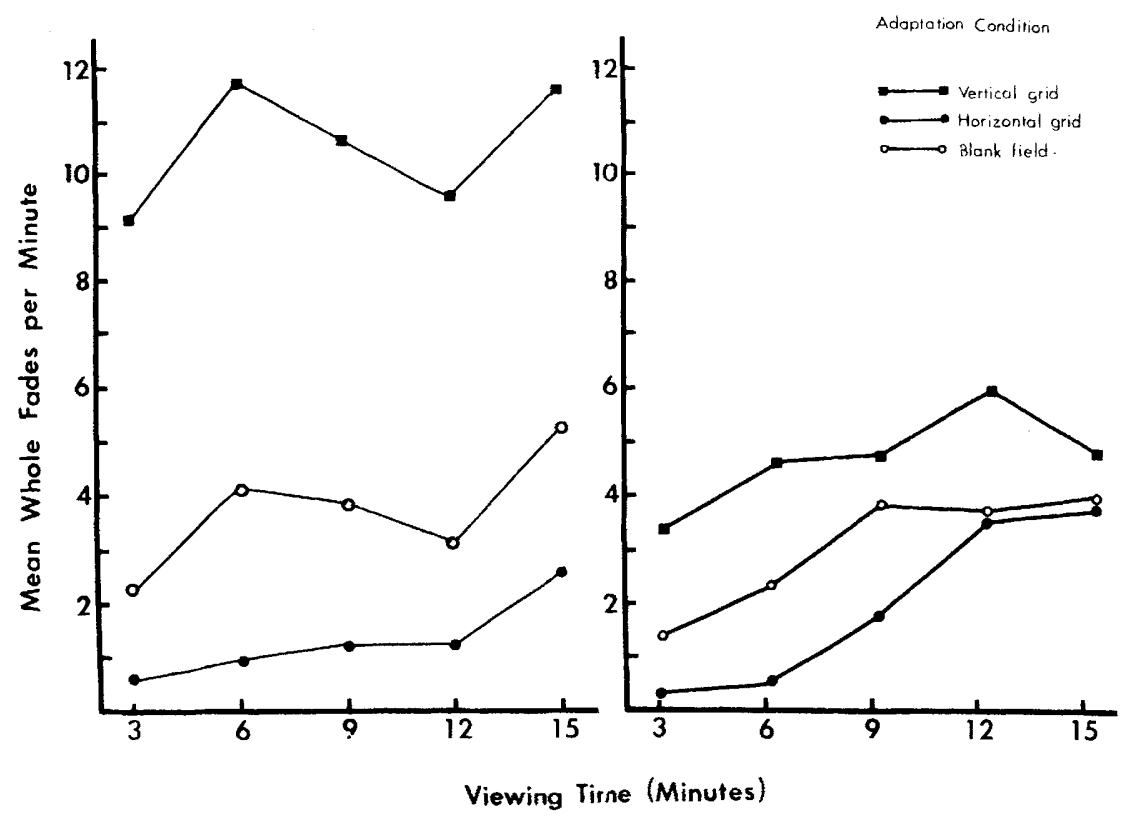

Fig. 2. Frequencies of whole fades of a stabilized vertical line following different nonstabilized adaptation conditions for monoptic (left panel) and dichoptic (right panel) viewing. Each data point represents the mean frequency with which the test line faded during a $3-\mathrm{min}$ interval of the $15-\mathrm{min}$ viewing session.

$2.0 \mathrm{fL}$ by means of neutral density wedges located in each channel and maintained by continuous monitoring of filament current at each source lamp.

\section{PROCEDURE AND}

EXPERIMENTAL DESIGN

Six experimental conditions were defined by combining the three types of adapting field-the horizontal grating, the vertical grating, or the patternless field-with monoptic and dichoptic viewing conditions. Each $\mathrm{O}$ was tested under all conditions twice, with conditions assigned randomly to 6 days of testing for each $\mathrm{O}$.

Each experimental session consisted of an adaptation phase in which the $O$ viewed the adaptation field for $15 \mathrm{~min}$ in nonstabilized Maxwellian view and a test phase in which the test line was viewed for $15 \mathrm{~min}$ in stabilized Maxwellian view. Under monoptic viewing conditions, the left eye was both adapted and tested; under dichoptic viewing conditions, the right eye was adapted and the left eye was tested.

Prior to the adaptation phase, the $\mathrm{O}$ inserted the contact lens and assumed the appropriate position for nonstabilized viewing. The adaptation field was then presented through Channel 1. Following the adaptation interval, the $\mathrm{O}$ shifted position to obtain stabilized viewing of the test field which was presented through Channel 2. Transitions between nonstabilized and stabilized viewing positions required a few seconds.
Responses to the stabilized test pattern were recorded by means of two telegraph keys under the O's left hand. During the test phase of each session, two categories of response were recorded, whole fades and partial fades. The $O$ responded to a whole fade whenever the entire stimulus was faded from view by pressing the appropriate key. A partial fade was recorded when any part, but not all, of the line was faded from view. Wholeand partial-fade frequencies (in fades/minute) were obtained from a two-channel event recorder which was activated by the telegraph keys.

RESULTS AND DISCUSSION

In past studies, we have found that the frequency of partial fades is relatively insensitive to pattern effects. Statistical tests on the partial-fade frequencies for these data reconfirm that result in that no significant effects occurred when partial fades were analyzed by analysis of variance procedures. Accordingly, the whole-fade frequencies are presented in Fig. 2 for all conditions of viewing. Under both monoptic (left panel) and dichoptic (right panel) conditions, relatively large and consistent differences were created by adaptation conditions. An analysis of variance of the 2 (Os) by 3 (adaptation conditions) by 5 (durations of test viewing) design yielded a significant adaptation condition main effect for both monoptic $(F=29.72 ; \mathrm{df}=2,2$; $\mathrm{p} \leqslant .05)$ and dichoptic $(\mathrm{F}=34.66 ; \mathrm{df}$ $=2,2 ; p \leqslant .05$ ) viewing. These data are very similar to previous data. When adaptation is to an unpatterned field, the time course and level of fading were very similar to previous data when no adaptation preceded testing (Cosgrove et al, 1972). Adaptation to a vertical grating increased the fading rate of a vertical test line; the horizontal grid suppressed the fading rate (Schmidt et al, 1972).

It is generally known that prolonged viewing of patterned stimuli produces pattern-specific aftereffects. Campbell \& Kulikowski (1966) have reported orientation-specific masking effects following pattern adaptation. Aftereffects may also include a frequency-specific contrast threshold elevation in periodic patterns (Blakemore \& Campbell, 1969) and orientation-specific chromatic aftereffects (Fidell, 1970). The attempt is commonly made to relate $\mathrm{these}$ aftereffects to neurophysiological findings in which single cells are selectively tuned to particular patterns and orientations (e.g., Hubel \& Wiesel, 1965, 1968). Our data demonstrate that prolonged viewing reliably influences whole-fade rates of stabilized stimuli in a manner most parsimoniously explained in terms of adaptation of orientation-specific mechanisms.

We have argued in more detail elsewhere that the organized character of the fading of stabilized images reflects the selective adaptation of the population of central analyzers for that particular stimulus (Schmidt, Cosgrove, \& Brown, 1972). Moreover, we have also argued that the suppression of fading of a vertical test line following prolonged adaptation to a vertical grid and the enhancement following adaptation to a horizontal grid can be explained in terms of the described behavior of higher order hypercomplex cells (Hubel \& Wiesel, 1965). The fact that these effects can be obtained dichoptically may be explained by the fact that it is known that a large proportion of cortical pattern processing cells are binocularly driven.

\section{REFERENCES}

BLAKEMORE, C., \& CAMPBELL, F. W. On the existence of neurones in the human visual system selectively sensitive to the orientation and size of retinal images. Journal of Physiology, 1969, 203, 237-260.

BROWN, D. R., SCHMIDT, M. J. FULGHAM, D. D., \& COSGROVE, M. P. Human receptive field characteristics: Probe analysis of stabilized images. Vision Research, in press.

CAMP BELL, F. W., \& KULIKOWSKI, J. J. Orientational selectivity of the human visual system. Journal of Physiology, 1966, 187, 437-445.

CLOWES, M. B., \& DITCHBURN, R. W. An improved apparatus for producing a stabilized retinal image. Optica Acta, $1959,6,252-265$.

COSGROVE, M. P., SCHMIDT, M.J., 
FULGHAM, D. D., \& BROWN, D. R. The dependent variable in stabilized retinal image studies. Vision Research, 1971, 11, 1183-1187.

COSGROVE, M. P., SCHMIDT, M. J., FULGHAM, D. D., \& BROWN, D. R. Stabilized images: Dependent variable specificity of pattern-specific effects with prolonged viewing. Perception \& Psychophysics, 1972, 11, 398-402.

FIDELL, L. S. Orientation specificity in chromatic adaptation of human "edge-detectors." Perceptio

Psychophysics, 1970, 8, 235-237.

HUBEL, D. H.. \& WIESEL, T. N. Receptive fields and functional architecture in two non-striate visual areas (18 and 19) of the cat. Journal of Neurophysiology, 1965 , 28, 229-289.
HUBEL, D. H., \& WIESEL. T. N. Receptive fields and functional architecture of monkey striate cortex. Journal of Physiology, 1968, 195, 215-243.

SCHMIDT, M. J., COSGROVE, M. P., \& BROWN, D. R. Functional relationships among populations of orientation-specific mechanisms in the human visual system. Perception \& Psychophysics, 1972, 11, 389-392.

SCHMIDT, M. J., FULGHAM, D. D., \& BROWN, D. R. Stabilized images: The search for pattern elements. Perception \& Psychophysics, 1971, 10, 295-299.

$$
\text { NOTE }
$$

1. Each channel includes a tungsten ribbon filament source lamp (So), lens train, target slide, and a stepping motor-operated shutter. Axes of both channels are congruent after passing through Beamsplitter $\mathrm{BS}_{1}$. The axis of interest then passes undeflected through Beamsplitter $\mathrm{BS}_{2}$, Lens $\mathrm{L}_{7}$, and onto Contact Lens Mirror CLM. Light is reflected back from CLM back through $L_{7}$ and into the viewing eve by means of $\mathrm{BS}_{2}$, Pentaprism PP, Lens $L_{8}$, and Mirrors $M_{1}$ and $M_{2}$. The reflected beam from CLM is deflected by an angle of $2 \theta$ for every angle $\theta$ of eye movement: in order to achieve stabilization, the image is, therefore, viewed through a distance twice that of the projection distance from CLM. Lenses $L_{7}$ and $L_{8}$ constitute a half-power telescope; $L$, produces a source image at the pupil plane; the lens of the viewing eye forms a target image on the retina. Thus, the requirements for Maxwellian view are satisfied. 